\title{
A Comparatively Analysis and Performance of Logical Topologies of Embedded Hypercube (LTOEH) Interconnection Network
}

\author{
Mohd. Kalamuddin Ahmad \\ Research Scholar \\ Department of CS \\ Mewar University, India
}

\author{
Mohd. Husain, PhD \\ Department of CS, \\ APJ Tech. University, Lucknow, India \\ (Formerly UP Tech. University, Lucknow)
}

\begin{abstract}
This paper is considered an important issue in the design of PE-Interconnection networks for massively parallel computing scalability. A detailed analysis shows that the double loop hypercube DLH $(\mathrm{m}, \mathrm{d})$ network is compared with mesh and torus embedded hypercube, torus embedded hypercube a better interconnection network in the properties of small diameter and highly connectivity, simple routing, scalability, constant node degree of topology, and the performance of communication. Show how to design the good interconnection network in parallel Architecture for less density as well as diameter to route the data to different path. Also it has been proved with the computational results of entire system that the embedded hypercube interconnection networks build is highly scale up in terms of communication. A complete design analysis and comparison of network with various other networks is given using different network parameters optimal of torus architecture rather than mesh architecture.
\end{abstract}

\section{Keywords}

Hypercube network, Topology, DLH, Data routing path, Embedded network, Network parameters and Scalability, Network metrics.

\section{INTRODUCTION}

Solve a given problem more rapidly, or to enable the solution of a problem that would otherwise be impracticable by a single worker. The way nodes are connected to one another nodes in the machines, in direct network architecture, each node has a point to point, or direct, connection to some number of nodes, called the neighboring nodes. Direct network have become popular architecture for constructing massively parallel computers because they scale well, that is the number of nodes in the system increases as well as communication bandwidth and processing capability of the system also increase [1], [3], and [11].

In direct network architecture, neighboring nodes may send packets to one another directly, while nodes that are not directly connected must rely on other nodes in the network to transfer packets from source to destination. Although a router's function could be performed by the corresponding local processor, dedicated routers are used to allow overlapped.

Computation as well as communication within each node, router supports some number of input and output channels. Internal channels connect the local processor memory to the

router. External channels are used for communication between routers and nodes. By connecting the input channels of one node to the output channels of other nodes, the topology of the direct network will be defined. For topologies in which packets may have to traverse some intermediate nodes, the routing algorithm determines the path selected by a packet to reach its destination. At each node, the routing algorithm indicates the next channel to be used. Efficient routing is critical to the performance of interconnection network [1], [6], [7], [9], [10], and [12].

In this paper the proposed work, how to route the data in massive parallel computer architecture with respect to reduce the diameter.

\section{PROPERTIES OF EMBEDDED NETWORKS}

The interconnection network is a vital role in a parallel processing. A good interconnection network is expected to have least number of links, topological network cost and more reliable. The interconnection network must be able to built scale up .The data routing functions in embedded hypercube network could be analyzed [3],[4,[5],[9] , [11].and [12].

\subsection{Mesh embedded}

A Single stage recirculating represent network. In network, each PE $i$ is allowed to send to any one of PE i+1, PE i-1, PE $i+r$ and PE i-r, Where $r=N$ in one circulating steps through entire network [5], [8], and [11].

The following routing function from (1) to (5) apply on simple mesh network [8] -

$$
\begin{aligned}
& R_{+1}(i)=(i+1) \bmod N \\
& R_{-1}(i)=(i-1) \bmod N \\
& R_{+r}(i)=(i+r) \bmod N \\
& R_{-r}(i)=(i-r) \bmod N \\
& R_{C}\left(k_{n-1} \ldots . . k_{d+1} k_{d} k_{d-1} \ldots . . k_{0}\right) \\
& =\left(k_{n-1} \ldots . . k_{d+1} k^{{ }_{d}} k_{d-1} \ldots k_{0}\right)
\end{aligned}
$$

Where $0 \leq \mathrm{i} \leq \mathrm{N}-1$, Commonly $\mathrm{N}$ know as perfect Square. 


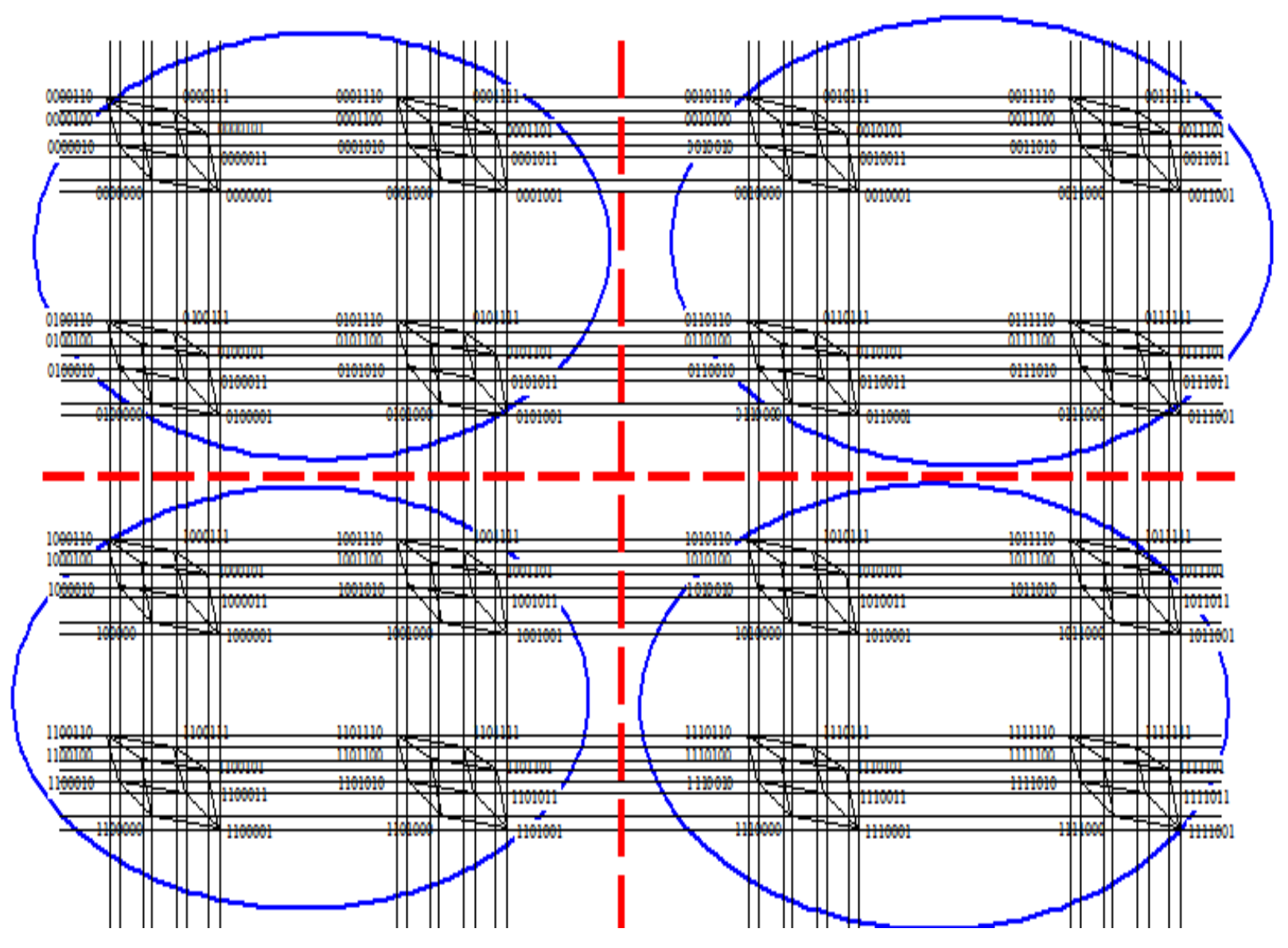

Figure: 1. $(4,4,8)$ Mesh Embedded Architecture

\subsection{Torus embedded}

Now suppose consider $\mathrm{a} \times \mathrm{b}$ be the size of some concurrent torus networks with a number of rows and $b$ number of columns and $\mathrm{N}$ being the number of nodes connected in the hypercube, the torus embedded hypercube network can be designed with the size of $(a, b, N)$. Nodes with identical positions in the torus networks will be a group of $\mathrm{N}$ number of nodes connected in the hypercube configuration and can be addressed with three parameters such as row number i, column number $\mathrm{j}$ of torus and address of node $\mathrm{k}$ in hypercube where the addressed node is residing. Hence, $\mathrm{a}(\mathrm{a}, \mathrm{b}, \mathrm{N})$-torus embedded hypercube network will have $\mathrm{a} \times \mathrm{b} \times \mathrm{N}$ number of nodes and a node with address as $(\mathrm{i}, \mathrm{j}, \mathrm{k})$ where $0 \leq \mathrm{i}<\mathrm{a}, 0 \leq \mathrm{j}$ $<\mathrm{b}$ and $0 \leq k<N$. The data routing functions of torus embedded hypercube as follow-

$$
\begin{aligned}
& R_{1}(i, j, k)=(i,(j+1) \bmod b, k) \\
& R_{2}(i, j, k)=(i,(b+j-1) \bmod b, k) \\
& R_{3}(i, j, k)=((i+1) \bmod a, j, k) \\
& R_{4}(i, j, k)=((a+i-1) \bmod a, j, k)
\end{aligned}
$$

And also used hypercube routing function equation ( 5 ) as above mentioned in the mesh embedded. 


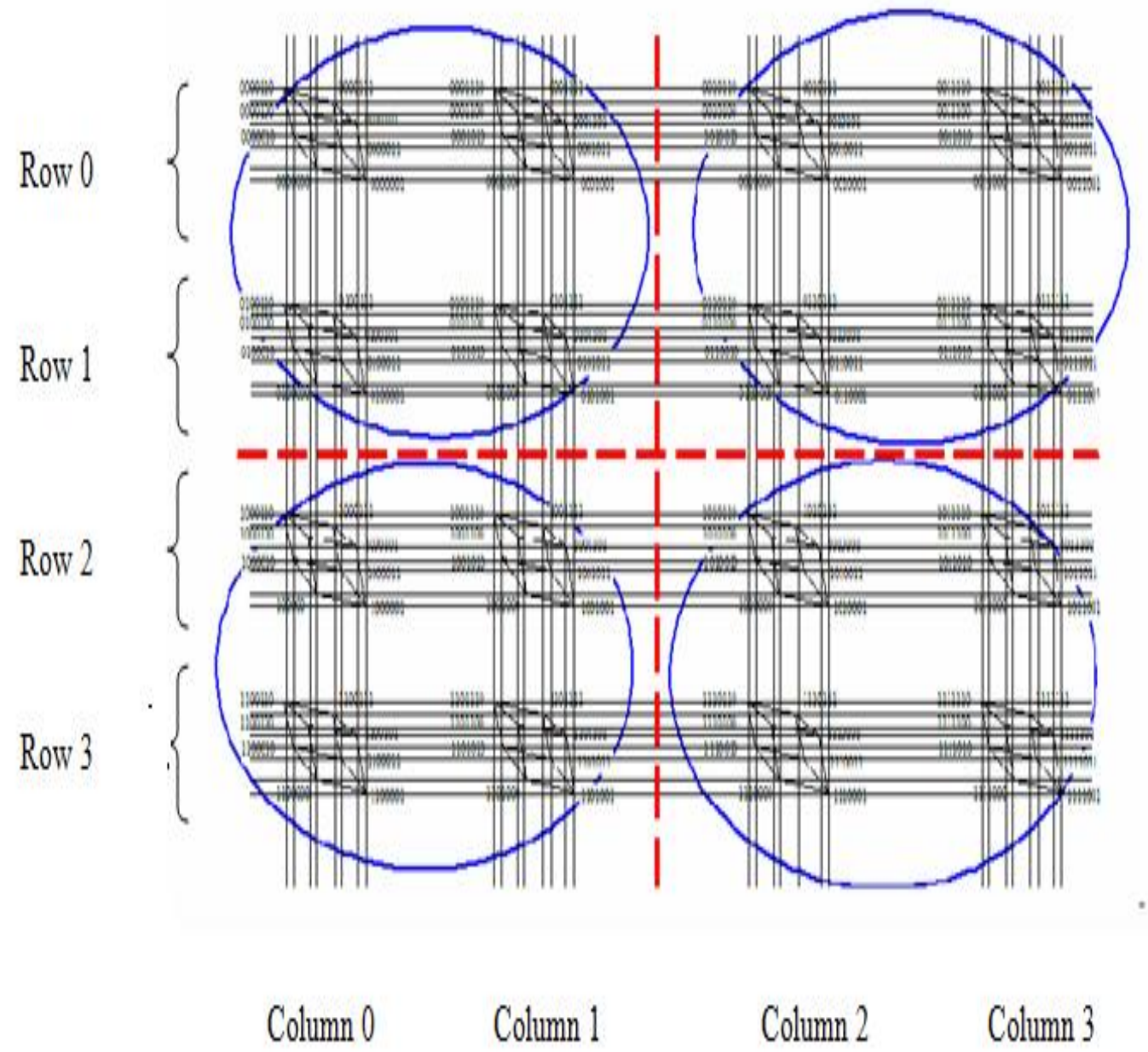

Figure: 2. (4, 4, 8) Torus Embedded Hypercube Network

The end to end connections of row and column of each connected in torus but are not represent in Figure 2. A wraparound connection is making along each row or column if

\subsection{Double loop embedded hypercube}

The total number of nodes in the DLH $(m, d)$ is $4 \mathrm{~m} \times 2 \mathrm{~d}$. When $\mathrm{m}=2$, the $\operatorname{DLH}(\mathrm{m}, \mathrm{d})$ can be constructed by combining the positive features of the hypercube topology, such as small diameter, high connectivity, symmetry and simple routing, and the scalability and constant node degree of the DL $(2 \mathrm{~m})$ topology as follows [2],[14], and [15]:

1) The $2^{d}$ nodes can be connected to be a $d$ dimensional hypercube network according routing equation (5), where $\mathrm{d}$ is denoted the dimensional hypercube networks.

2) The $4 \mathrm{~m}$ such kinds of $\mathrm{d}$ dimensional hypercube networks can be divided into $2 \mathrm{~m}$ groups, in which any group can be coded with a group-id, which adopts Johnson code from 0 to $2 m$, and any $d$ they have equal label as a complete of torus embedded hypercube network $(4,4,8)$.

dimensional hypercube network in a same group can also be coded with a net-id using 0 or 1 .

3) The nodes with both the same node-id in different groups can be connected to a DL $(2 m)$ according to $d$.

4) The code of nodes in the DLH (m, d) when there is just one bit different between any two nodes, there will exist a link between them, that is to say, these two nodes are neighboring to each other.

Figure 3. Illustrate a DLH $(m, d)$ interconnection where solid lines represent hypercube links and thin lines represent 


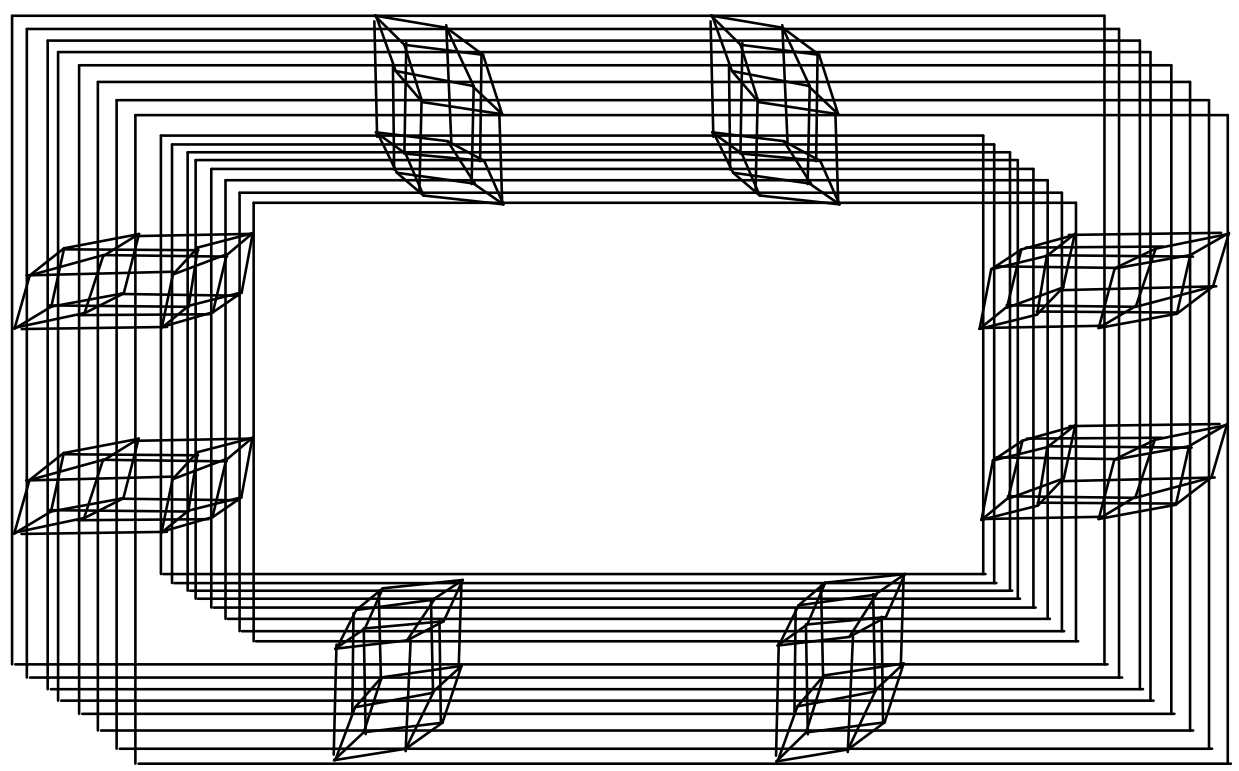

Figure: 3. Double Loop Embedded Hypercube

DL (2m) links.. The size of the DLH(m,d) can grow without altering the number of links per node by expanding the size of the TL $(2 \mathrm{~m})$; for example, by adding four three-cubes on the perimeter of the $\operatorname{TL}(2 \mathrm{~m})$ in figure . A DLH $(4,3)$ consists of $4 \times 4 \times 23=128$ nodes. It can be viewed as eight concurrent DL
$(2 \mathrm{~m})$ where eight nodes having identical DL ( $2 \mathrm{~m})$ addresses form one three-cube. Alternatively, it can be viewed as 16 concurrent three-cubes in which 16 nodes having identical hypercube addresses form a DL (2m).

\section{RESULTS AND DISCUSSION}

\subsection{Node degree}

Table-1 and figure 4, Gives the comparison of node degree as a function of the number of processors of various other popular networks for parallel computing system.

Table 1: Comparative Results of Node Degree, For Different Topology

\begin{tabular}{|c|c|c|c|c|c|c|c|}
\hline \multirow[t]{2}{*}{ Types of Network } & \multicolumn{7}{|c|}{ Number of Processors } \\
\hline & 128 & 256 & 512 & 1024 & 2048 & 4096 & 8192 \\
\hline Ring & 2 & 2 & 2 & 2 & 2 & 2 & 2 \\
\hline Linear Array & 2 & 2 & 2 & 2 & 2 & 2 & 2 \\
\hline 2D-Mesh & 4 & 4 & 4 & 4 & 4 & 4 & 4 \\
\hline Illiac-Mesh & 4 & 4 & 4 & 4 & 4 & 4 & 4 \\
\hline 2D-Torus & 4 & 4 & 4 & 4 & 4 & 4 & 4 \\
\hline Hypercube & 7 & 8 & 9 & 10 & 11 & 12 & 13 \\
\hline k-ary-n Cube & $14, \mathrm{k}=2$ & $16, \mathrm{k}=2$ & $18, \mathrm{k}=2$ & $20, \mathrm{k}=2$ & $22, \mathrm{k}=2$ & $24, \mathrm{k}=2$ & $26, \mathrm{k}=2$ \\
\hline
\end{tabular}

The comparison indicates that the hypercube and k-ary n-cube interconnection networks are more expensive used in parallel computer, and it's not suitable for parallel architecture. For these two networks, the node degree increases drastically as the system expansion takes place. Obviously the cost per node also increases tremendously for these two networks as the system is scaled up shown in figure 4 . 


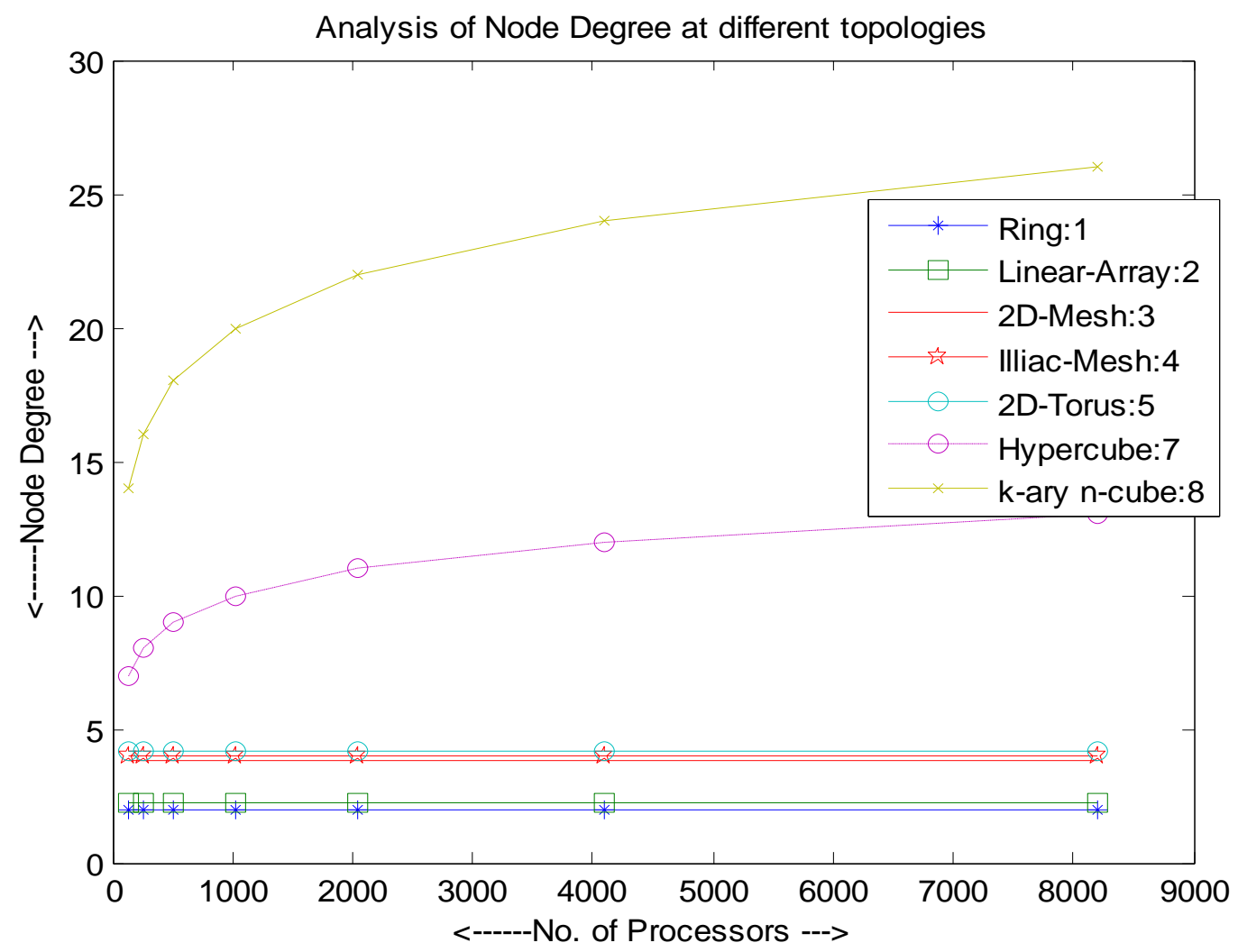

Figure: 4. Node degrees Analysis

\subsection{Total number of links}

Table 2: Comparative Results of Number of Links for Network Topology

\begin{tabular}{|c|c|c|c|c|c|c|c|}
\hline \multirow{2}{*}{ Types of Network } & \multicolumn{7}{|c|}{ Number of Processors } \\
\cline { 2 - 8 } & 128 & 256 & 512 & 1024 & 2048 & 4096 & 8192 \\
\hline Ring & 128 & 256 & 512 & 1024 & 2048 & 4096 & 8192 \\
\hline Linear Array & 127 & 255 & 511 & 1023 & 2047 & 4095 & 8191 \\
\hline 2D-Mesh & 234 & 480 & 980 & 1984 & 4006 & 8064 & 16203 \\
\hline Illiac -Mesh & 256 & 512 & 1024 & 2048 & 4096 & 8196 & 16384 \\
\hline 2D-Torus & 256 & 512 & 1012 & 2048 & 4096 & 8192 & 16384 \\
\hline Hypercube & 64 & 128 & 256 & 512 & 1024 & 2048 & 53248 \\
\hline k-ary-n Cube & $896, \mathrm{k}=2$ & $2048, \mathrm{k}=2$ & $4608, \mathrm{k}=2$ & $10240, \mathrm{k}=2$ & $22528, \mathrm{k}=2$ & $28672, \mathrm{k}=2$ & $1.06 \mathrm{e}+005$ \\
\hline
\end{tabular}

Table-2 and Figure 5 gives the comparison of Total number of wires or links for a PE-Interconnection network is expected to be in above table. In table 2 to reflects link complexity and directly related to the cost of the networks.

Table 2 shows that the number of links w.r.t. the design of simple network scaling in the parallel computing. It is observed that the total number of connection of two dimensional static topologies like linear array, 2D-mesh, 2Dtorus, and hypercube lies in between ring and k-ary n-cube. It is to be noted that the k-ary n-cube offers larger number of links network as illustrate in figure 5 . 


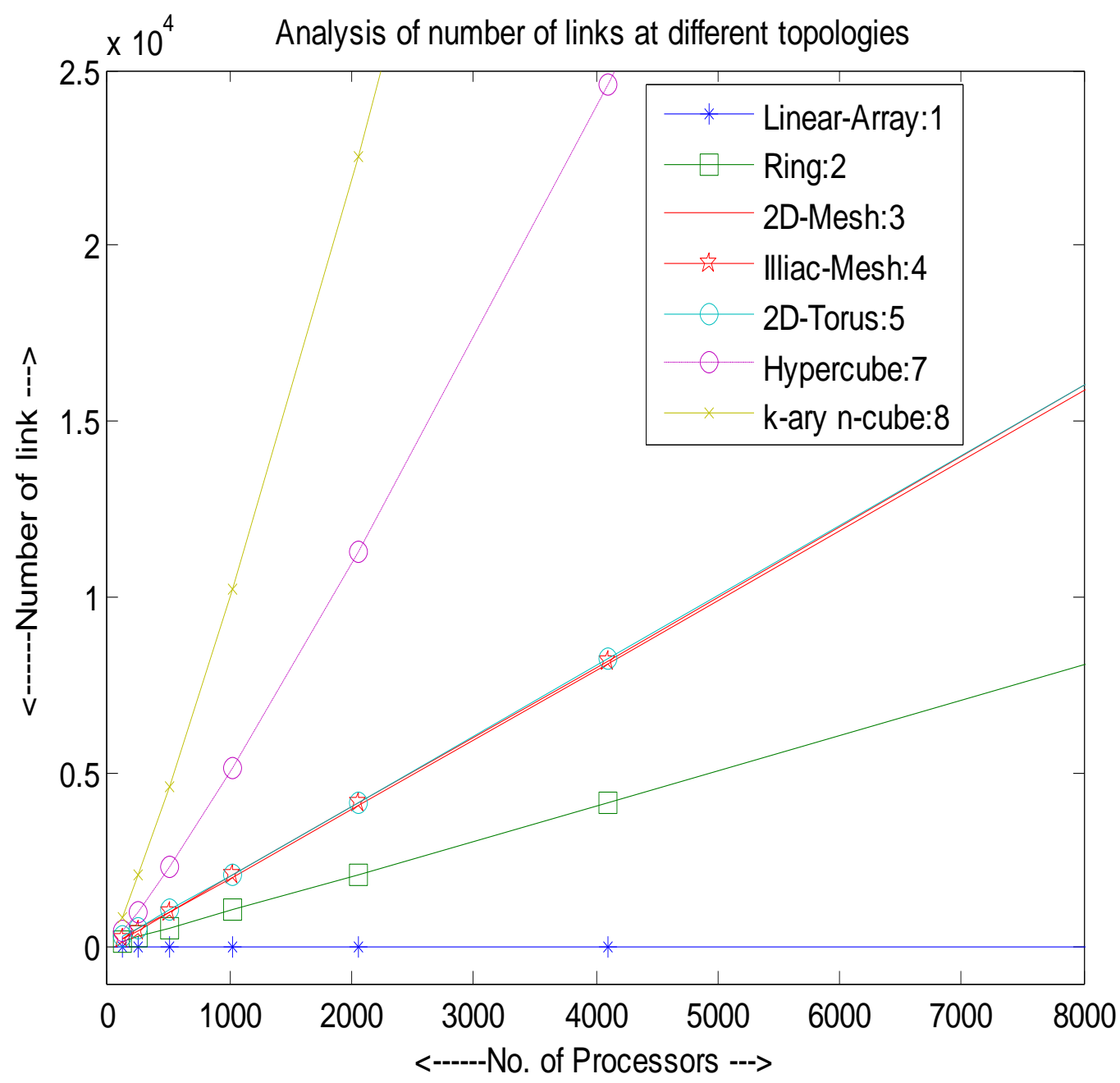

Figure: 5. Analysis of links

\subsection{Network diameter analysis}

Table- 3 and Figure 6 shows the comparison of network diameter at different topology, and also Table 4 and figure 7 shows the comparison of network as a function of the number of processors of various other popular networks along with the embedded hypercube network worth to mention that due to their bottleneck performance as per the last comparisons, in the table 3 indicates lower diameter of the topology are mesh, torus Iliac mesh and hypercube. Therefore lower diameter topologies are used in parallel computing for PEInterconnecting device but table 4 and figure 7 gives comparative analysis between mesh and torus embedded hypercube networks, mesh embedded hypercube are dropped because due to high diameter.

Table 3: Comparative Results of Diameter at different network topology

\begin{tabular}{|l|c|c|c|c|c|c|c|}
\hline \multirow{2}{*}{$\begin{array}{l}\text { Types of } \\
\text { Network }\end{array}$} & \multicolumn{7}{|c|}{ Number of Processors } \\
\cline { 2 - 8 } & 128 & 256 & 512 & 1024 & 2048 & 4096 & 8192 \\
\hline Ring & 64 & 128 & 256 & 512 & 1024 & 2048 & 4096 \\
\hline Linear Array & 127 & 255 & 511 & 1023 & 2047 & 4095 & 8191 \\
\hline 2D-Mesh & 21 & 30 & 43 & 62 & 89 & 126 & 1179 \\
\hline Illiac -Mesh & 10 & 15 & 22 & 31 & 44 & 63 & 90 \\
\hline 2D-Torus & 12 & 16 & 24 & 32 & 11 & 64 & 92 \\
\hline
\end{tabular}




\begin{tabular}{|l|c|c|c|c|c|c|c|}
\hline Hypercube & 7 & 8 & 9 & 10 & 11 & 12 & 13 \\
\hline k-ary-n Cube & $7, \mathrm{k}=2$ & $8, \mathrm{k}=2$ & $9, \mathrm{k}=2$ & $10, \mathrm{k}=2$ & $11, \mathrm{k}=2$ & $12, \mathrm{k}=2$ & $13, \mathrm{k}=2$ \\
& & & & & & & \\
\hline
\end{tabular}

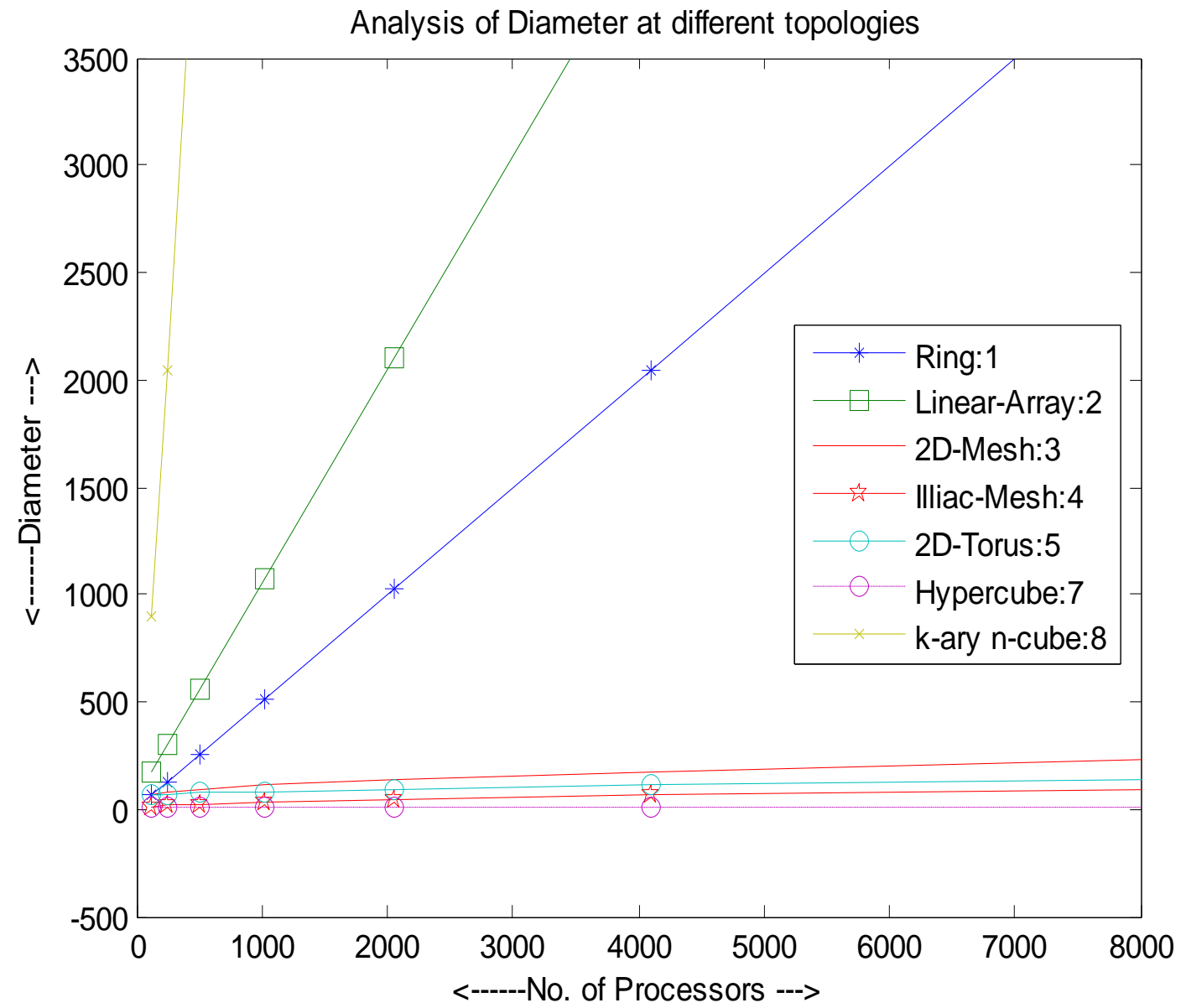

Figure: 6. Analysis of Diameter of different Topology

Table 4: Comparative Results of Diameter for Embedded Network

\begin{tabular}{|c|c|c|c|c|c|c|}
\hline \multirow[t]{2}{*}{ Types of Network } & \multicolumn{6}{|c|}{ Number of Processors } \\
\hline & 512 & 1024 & 2048 & 4096 & 8192 & 16384 \\
\hline$(8,8, \mathrm{~N})$ Mesh Embedded Hypercube & $17, \mathrm{~N}=8$ & $18, N=16$ & $19, \mathrm{~N}=32$ & $20, N=64$ & $21, N=128$ & $22, \mathrm{~N}=256$ \\
\hline $\begin{array}{l}(16,16, N) \text { Mesh Embedded } \\
\text { Hypercube }\end{array}$ & $31, \mathrm{~N}=2$ & $32, \mathrm{~N}=4$ & $33, \mathrm{~N}=8$ & $34, N=16$ & $35, \mathrm{~N}=32$ & $36, \mathrm{~N}=64$ \\
\hline $\begin{array}{l}(32,32, N) \text { Mesh Embedded } \\
\text { Hypercube }\end{array}$ & 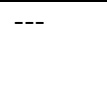 & $64, \mathrm{~N}=1$ & $65, N=2$ & $66, N=4$ & $67, N=8$ & $68, N=16$ \\
\hline$(8,8, \mathrm{~N})$ Torus Embedded Hypercube & $11, \mathrm{~N}=8$ & $12, N=16$ & $13, \mathrm{~N}=32$ & $14, N=64$ & $15, \mathrm{~N}=128$ & $16, \mathrm{~N}=256$ \\
\hline $\begin{array}{l}(16,16, N) \text { Torus Embedded } \\
\text { Hypercube }\end{array}$ & $17, \mathrm{~N}=2$ & $18, \mathrm{~N}=4$ & $19, \mathrm{~N}=8$ & $20, N=16$ & $21, N=32$ & $22, \mathrm{~N}=64$ \\
\hline
\end{tabular}




\begin{tabular}{|l|l|l|l|l|l|l|}
\hline $\begin{array}{l}\text { (32,32,N)Torus Embedded } \\
\text { Hypercube }\end{array}$ & --- & $32, \mathrm{~N}=1$ & $33, \mathrm{~N}=2$ & $34, \mathrm{~N}=4$ & $35, \mathrm{~N}=8$ & $36, \mathrm{~N}=16$ \\
\hline (m,d) Double Loop Hypercube, $\mathrm{d}=3$ & $20, \mathrm{~m}=16$ & $36, \mathrm{~m}=32$ & $68, \mathrm{~m}=64$ & $132, \mathrm{~m}=128$ & $260, \mathrm{~m}=256$ & $516, \mathrm{~m}=512$ \\
\hline
\end{tabular}

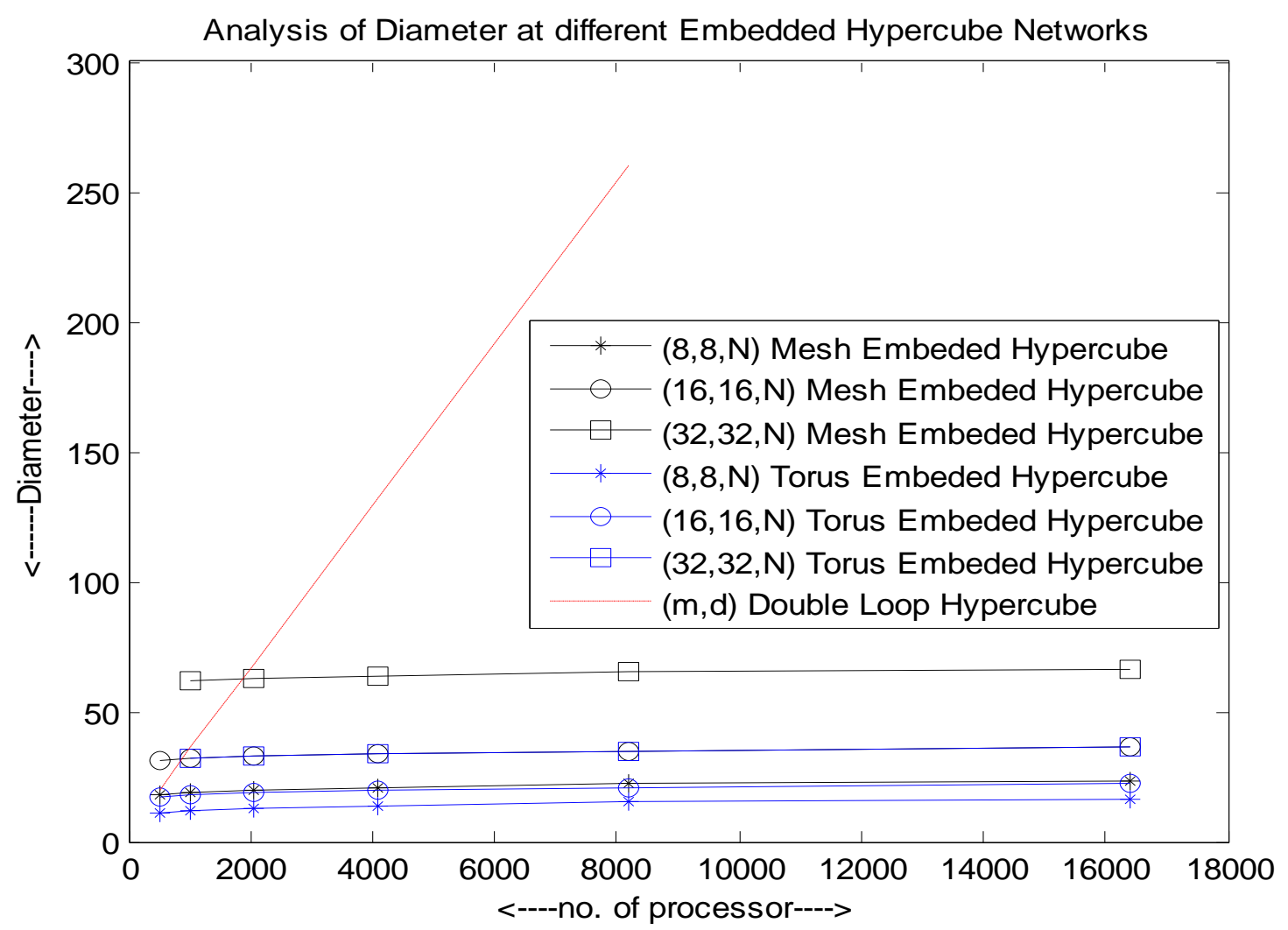

Figure: 7. Analysis of diameter

In the results of embedded architecture given in Table 4 and Figure 7 as far as the network diameter is concerned, the torus embedded hypercube network required needs minimum network diameter to get interconnected between a source node and a destination node.

We mentioned the required the total diameter from source to destination is-

Mesh D s-d = Diameter of two dimensional mesh + Diameter of hypercube

$=2(\sqrt{ } \mathrm{p}-1)+\log \mathrm{p}$

Where $\mathrm{p}$ is no. of processor in two dimensional mesh, and p' is no. of processor in hypercube.

We are also mentioned here Torus embedded hypercube diameter from source to destination-

Torus D s-d = Diameter of two dimensional torus + Diameter of hypercube

$$
=\sqrt{ } \mathrm{p}+\log \mathrm{p}
$$

The diameter of the Double loop hypercube DLH (m, d) [12] is-

Diameter of DLH $(m, d)=m+d+1$

Where $\mathrm{p}$ is no. of processor in two dimensional Torus, $\mathrm{p}$ ' is no. of processor in hypercube. (Here we mentioned the total number of processors in two dimensional mesh or torus embedded hypercube $\left.=\mathrm{p}+\mathrm{p}^{\prime}\right)$

In comparatively analysis -diameter of $(8,8, \mathrm{~N})$ mesh embedded hypercube system are same as $(16,16, \mathrm{~N})$ torus embedded hypercube system, and also diameter of $(16,16, \mathrm{~N})$ mesh embedded hypercube system are same as $(32,32, \mathrm{~N})$ torus embedded hypercube system, shown in figure 5.

Hence the torus embedded hypercube network is much better than mesh embedded hypercube network and double loop hypercube DLH ( $\mathrm{m}, \mathrm{d})$ as far as performance metrics and speed up than mesh embedded hypercube as well as Double Loop Hypercube.

\section{CONCLUSION}

This paper has considered a PE-Interconnection network is a combination of hypercube and torus network topologies. In this paper is discussing the node degree, no. of links, and diameter of static network without and with embedding of hypercube. The analysis of results provide an idea about that torus embedded hypercube interconnection network is highly scalable, this network structure provides a immense architectural support for massive parallel computing. The growth of the network is more efficient in terms of communication and speed up. The comparison shows that the $\mathrm{n}$-cube hypercube and $\mathrm{K}$-ary n-cube interconnection networks are expensive and not suitable for parallel architecture. For these two networks, the node degree increases dramatically as 
the system spreading out takes place. The number of links with respect to the scaling design of simple network. It is observed that the total number of connection for two Dimensional static network like Mesh, Torus, Illiac-Mesh, and Ring lies in between Linear array and k-ary n-cube

\section{REFERENCES}

[1] K. Hwang, Advanced Computer Architecture: Parallelism, Scalability, Programmability. New York McGraw-Hill, 1993.

[2] Kim Jong-Seok, Lee Hyeong-Ok and Heo Yeong-Nam, "Embedding among $\operatorname{HCN}(n, n), \operatorname{HFN}(n, n)$ and hypercube" , Proceedings of Eighth International conference on parallel and distributed systems (ICPADS)-2001 pp 533 - 540, 26-29 June 2001.

[3] A. Louri and H. Sung, "An Optical Multi-Mesh Hypercube: A Scalable Optical Interconnection Network for Massively Parallel Computing," IEEE J. Lightwave Technology, vol. 12, pp. 704-716, Apr. 1994.

[4] N. Gopalakrishna Kini, M. Sathish Kumar and Mruthyunjaya H.S., "A Torus Embedded Hypercube Scalable Interconnection Network for Parallel Architecture," IEEE explore conference publications, Mar.2009, pp.858-861.

[5] Ahmed Louri and Hongki Sung, "An Optical Multi-Mesh Hypercube: A Scalable Optical interconnection Network for Massively Parallel Computing," Journal of Light wave Technology, Vol.12, No.4, Apr.1994, pp.704-716.

[6] Ahmed Louri and Hongki Sung, "An OpticalMultiMeshHypercube:AScalableOpticalinterconn ection Network for Massively Parallel Computing," Journal of Light wave Technology, Vol.12, No.4, Apr.1994, pp.704-716.

[7] L.M. Ni, and P.K. McKinley, "A Survey of Wormhole Routing Techniques in Direct Networks", IEEE Computer, vol.26, no.2, pp. 62-76, Feb. 1993.

[8] M. K. Ahamad, M. Husain.” Required Delay of Packet Transfer Model for Embedded Interconnection Network" International Journal of Engineering Research \& network. It is preferred to have a network with a network diameter of minimum value. The result of comparison shows that Torus and mesh network, mesh has the large network diameter. Further, for this network, the network diameter increases tremendously as the system is scaled up.

Technology (IJERT) Vol. 2 Issue 1, January- 2013 ISSN: 2278-0181.

[9] M. K. Ahamad, M. Huasain, A.A.Zilli ,"A Statistical Analysis and Comparative Study of Embedded Hypercube ", International Journal of Computer Applications (0975 - 8887) Volume 103 - No 16, October 2014

[10] G.M. Chiu, "The Odd-Even Turn Model for Adaptive Routing,"IEEE Trans. Parallel and Distributed Systems, vol. 11, no. 7, pp. 729738, July 2000., No.11, Nov.1994.

[11] W.J. Dally, "Performance Analysis of k-ary n-cube Interconnection Networks," IEEE Trans. Computers, vol. 39, no. 6, pp. 775-785, June 1990.

[12] Zhaoyang Li, Yi Zhang, Yu Chen and Ruichun Tang, "Design and implementation of a high-performance interconnection network," Proceedings of the Fourth International Conference on Parallel and Distributed Computing, Applications and Technologies, 2003, PDCAT'2003, 27-29 Aug.2003, pp.17-20.

[13] Hesham El-Rewini and Mostafa Abd-El-Barr, "Advanced Computer Architecture and Parallel Processing," John Wiley \& Sons, Inc., Hoboken, New Jersey, 2005.

[14] Ahmed Louri and Hongki Sung, "A scalable optical hypercube-based interconnection network for massively parallel computing," Applied optics, Vol.33.

[15] Y. Saad and M.H. Schultz, "Data Communication in Parallel Architectures," Parallel Computing, vol. 11, pp. 131-150, 1989.

[16] S.L. Johnsson and C.T. Ho, "Optimum Broadcasting and Personalized Communication in Hypercubes", IEEE Trans. Computers, vol. 38, no. 9, pp. 1249-1268, 1989. 